\title{
Vrudh Sevak: A Digital Assistant for Lonely Elders using 'Technology of Device Connectivity'
}

\author{
Vinaya $B^{1}$, Dr. M. C. Padma ${ }^{2}$ \\ M. Tech $2^{\text {nd }}$ Year, CS\&E, PESCE, Mandya, India ${ }^{1}$ \\ Professor and Head, CS\&E, PESCE, Mandya, India ${ }^{2}$
}

\begin{abstract}
According to a report from Times of India, about 15 million old age people are living alone in India. For various reasons they are living alone and its probability is high in cities. Since they are old, their life is limited to a bound, loneliness and will be having health issues. Old age contributes forgetfulness in many, poor health support and loneliness due to various reasons. We proposed an assistance (Vrudh Sevak) to the majority of the problem faced by the lonely elders using 'Internet of Things' and smart phone technology. Internet of things is a propelled worldview to interface physical and virtual substances for improved administrations. We proposed different methodologies to address the various issues such as forgetfulness using smart phone and its voice based assistance, health care by IOT sensors, social communication among same age group and connecting to concerned people via GSM technology. We also proposed a way to improve their health and habit based on the user information.
\end{abstract}

Keywords: Vrudh Sevak, IOT, GSM, Smart Phone

\section{INTRODUCTION}

According to Times of India Report dated 2014 October 1st, it's found that 15 million people are living alone. Loneliness is more common in the developed and developing countries. It's not even considered as the biggest problem, where in society falls into an imbalance state. Usual reasons we could expect are spouse death, divorcee, and children's moved to other place for their carrier or future. These problems cannot be solved under the court of law or under any legislation. These are the human sentimental and feeling issues. It's all the effect of modernization and cannot be stopped. But we can sort out some of the major problems faced by the lonely elders which screw their mental and physical health. Which evokes to give solutions for them.

This is one of the motivation for us take to up the work and progress on it. Usual Problems of the lonely elders are lack of memory, Sudden and irregular health variation, need someone - who care for their feelings and health advice. Above all they need a doctor or a concerned people in contact when its health emergency or in any other emergencies. Considering forgetfulness problem, for example while cooking elder may concentrate on the other things and forget about the cooking. This causes the waste of fuel and food. Additionally, if they are going outside they may forget wallet, Walking stick or mobile phones, kerchief, spectacles etc. Nowadays we can find 6 in 10 people who are having some kind of medical issues and under medication. So it's not trivial to guess old age people 9 out of 10 are under medication. Usual problem with this is they may forget to take medication in time. Obliviously we need some external support to handle this kind of situations.

One of the most cautious and precious thing after Breath is Health. People of elderly need frequent care nowadays due to various diseases. Usual problems are High body temperature or low temperature, Heart related issues and sugar complaints. During health emergency obviously they need basic care at least from doctors. All though when we consider the mentioned issues are majorly faced and important to sort out, these are just usual physical issues that contributed by old age in one or the other way. Main leader for all these things is Mental Health. If the people are strong enough mentally, at least they can face the problem with enough confidence. How one could get mental happiness in old age just from all materialistic things? They need an ears to hear their grief and to share their happiness. How can we expect such things can be so easy when family apart, place apart and in modern civilization? Also who we will sit and talk so much freely that too in the modern competitive world? If it can be, then it can be another elder who may or may not be lonely. Habits are considered to be the one which makes us happier than our usual routine work. So it's good if elder get some new and detailed information about their habits, which updates and makes them happy. Last but not least, what we eat is also more important which decides the health factor. Considering all the above points mentioned, to give the solution to the mentioned routine problems we found IOT is the best thing, using which we can 
solve their problems up to few levels. Internet of things is an emerging technology which integrated other embedded system into it and contributes to the specific and useful goal to be achieved. Also SMART PHONE is one the wide spreading and fast growing equipment which almost covered that world. Using smart phones we offered social communication, helpful habit and health suggestion tips. We deployed Reseases Microcontroller based IOT systems with GSM enabled. So that drug reminder can be done via caretaker just by setting the required time. We offered embedded module to get Health Parameters and detect any problems early and to take necessary actions. We used one more model called SLIPPER unit which addresses the forgetfulness. Also used IOT sensors to detect gas and fire accidents. Considering the IOT technologies used, we can say that it's cost effective, easily configurable, reconfigurable and maintainable.

\section{LITERATURE SURVEY}

We have undergone various research journals and articles regarding the work carried in the proposed system. And those are explained as follows.

Authors - "Atika Arshad, Ahmad Fadzil Ismail, Sheroz Khan, Wahidah Hashim and Mohammad Kamrul Hasan" was proposed and implemented a system in order to monitor sleeping pattern of an elderly and this is the key to measure the health conditions. Possibly - restless sleep, unusual changes in the scheduled activities, typical changes in the routine activities. Also proposed that the system could able to check availability of the person on the bed, in and out schedules of the bed. It adds smart lightning to the elderly bedrooms or wherever required. Authors likewise referenced that the framework is minimal effort since it depends on capacitive detecting. They likewise referenced that framework is effectively mountable in the interim another sensor can be included and evacuated whenever. The executed framework can store a client's physiological information for a whole day, additionally for seven days. As per Authors - there is one noteworthy disadvantage that the proposed framework can be just actualized in raised deck homes for framework to be inconspicuous. It's observed that proposed system mainly focused on the bedroom monitoring of an elderly and his/her difference in the routine life is the key to measure the health variations. In our system we used the available IOT sensor for the accurate measurement of the health conditions and made life easy in the entire homing [1].

Authors - "Hyun-Sik Kim, Jong-Su Seo, and Jeongwook Seo" presented an intelligent routine life monitoring model for realizing internet of things- assisted living for the lonely elders. Lonely elders common issues, for example, experience the ill effects of family issues, low pay, and troublesome life driving conditions. Daily monitoring system was pictured to be able to collect the daily activity information of an elderly from the emergency gateway. This is accomplished in collaboration with contactless activity sensors and help trigger. The framework profoundly gets information about information gathered, investigations and reports, ordinary and surprising examples of movement characterized in reference to the action information inferred utilizing the predefined action list. The normal movement data can be utilized to quantify and maintain a strategic distance from startling crisis circumstances, for example, abrupt passings. This proposed system can be utilized in various health and wellness applications. Though we have accuracy algorithms to identify the wellness using the daily routine life data, we need to check exact medical conditions in order to derive an implication. This is the place where system is lagging. We used modern IOT based techniques to give accurate inferences [2].

Iman Azimi, Amir M. Rahmani, Pasi Liljeberg, Hannu Tenhunen - proposed an idea or big picture of what an elderly needed in the modern life style. Different methodologies have been as of late given to address the everyday life necessities of older. But there exists an absence of client focused examination in the proposed frameworks. Proposed system tells about the studies at the state of the art mean while the necessary requirement of the target system. Based on the lonely elders monitoring approaches to identify their pros and cons from a wide verities of viewpoints. Also the Proposed system introduces the modern categories and hierarchical model to elderly monitoring in-order to investigate objectives and challenges in a first to last fashion or also called as top down fashion. With every one of these things this examination builds up a general perspective on the region and talks about the current arrangements. It introduces the fundamental objectives and current patterns so the IOT advancements based framework can accommodate future observing frameworks [3].

Authors - "V. Mighali, L. Patrono, and M. L. Stefanizzi, Joel J. P. C. Rodrigues, Petar Solic" proposed a system focused on the old age people behavioural analysis through the IOT technologies. Which counteracts little Cognitive Impairment issues. Specifically, since movements and body motility are great signs to behavioural or body changes, mentioned work goal is to characterize a solid framework for controlling the position and the body motility of the senior individuals with minimal effort and low-control way. The system designed in order to complete "study of behavioural analysis and risk detection developed within the City4Age project", sponsored by the Horizon 2020 Programme of the European Commission [4]. This Research helped in IOT technologies embedding in wearable devices. This is one of the motivation for our slipper unit to implement. 


\section{SYSTEM ARCHITECTURE}

Overall system structure with its pictorial representation is depicted in the Fig.1. We integrated trending technologies 'android applications and Internet of things' to provide low cost, precise and easy maintaining system. Based on the usage and placement we divided system into 3 main units such as embedded, Slipper unit and smartphone unit. Each of them does its particular work when its turn on.

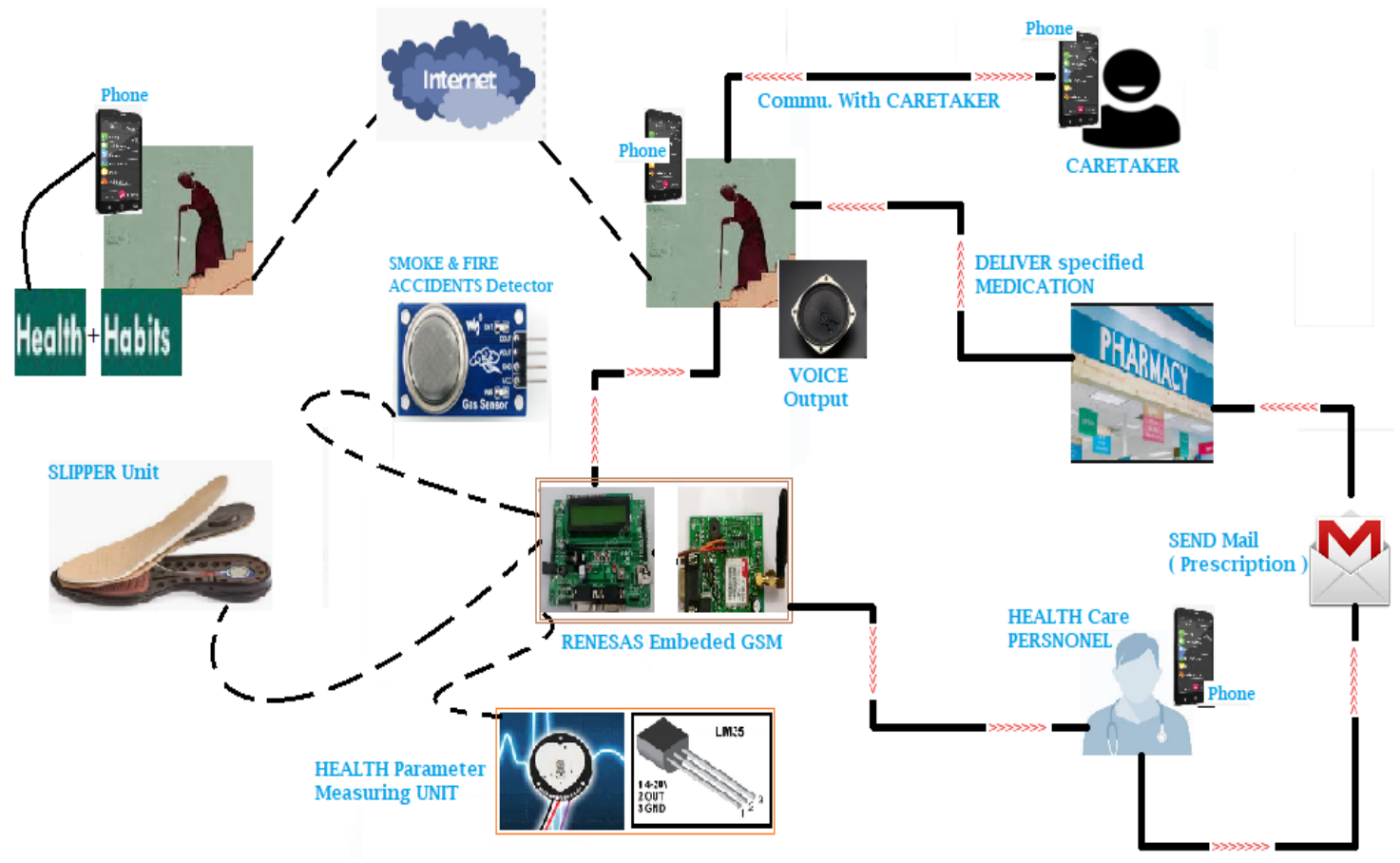

Fig. 1: Overall System Architecture

Health Parameter measuring unit measures details such as Heart Rate, Pulse and Body Temperature. If either of the body temperature or heart rate found to be above the specified range then it's treated as abnormal. The measured units are made available at the Healthcare personal. Healthcare personal will send an email of prescription to the specified drug outlets. Drug outlets will take the responsibility of delivery to the mentioned elder address.

Elder people usually has memory issue. So we created an android app for medication alarming. This app is used by caretaker, who will set the medication time in his app. Once the Android system time matches with this time then, a voice alarming is given to intimate the medication intake. Also when they are cooking if they busy with something else like TV, Mobile or anything like that then, we sense the smoke and intimate the Elder via continuous beep sound. The same technique is used to identify and alert elder when there is a fire accident. Slipper unit is also one module which is being used to address the problem of memory issue. Elder people may forget to take things while going outside. Things may include their spectacle, watch, mobile, walking stick, wallet etc. As soon as the elder touch the slipper, a voice output is going to take the responsibility of reminding things to be taken while going outside.

To establish social communication among elder we created an android app which facilitates chat among elder people. It's done via taking their name, age and clubbing into app of their age group. Also application takes input such as diseases, its condition and redirects into related videos which helps in better maintenance of the Health. Also its takes habit, height, weight and food-order to help in improving their habits. Renesas Controller is the core of the controlling system which executes and enables software instructions to co-ordinate with other devices. GSM system is used to intimate the caretaker, Health care personnel during fire accidents, health variations etc.

\section{SYSTEM MODULES}

In light of the utilization and situation we separated framework into 3 primary units, for example, embedded, Slipper unit and cell phone unit. Every one of them does its specific work when its turn on.

\section{A. Embedded unit}

Embedded unit is the focal unit, which comprises of microcontroller, GSM module, temperature sensor, smoke sensor, help keys, FN16MP playback gadget, speaker and a RF beneficiary (Rx). Android application will be introduced in the 
client's cell phone with android rendition 4.0 or above. Wellbeing Check Up unit comprises of sensors estimating for BP, Pulse and Heart Rate.

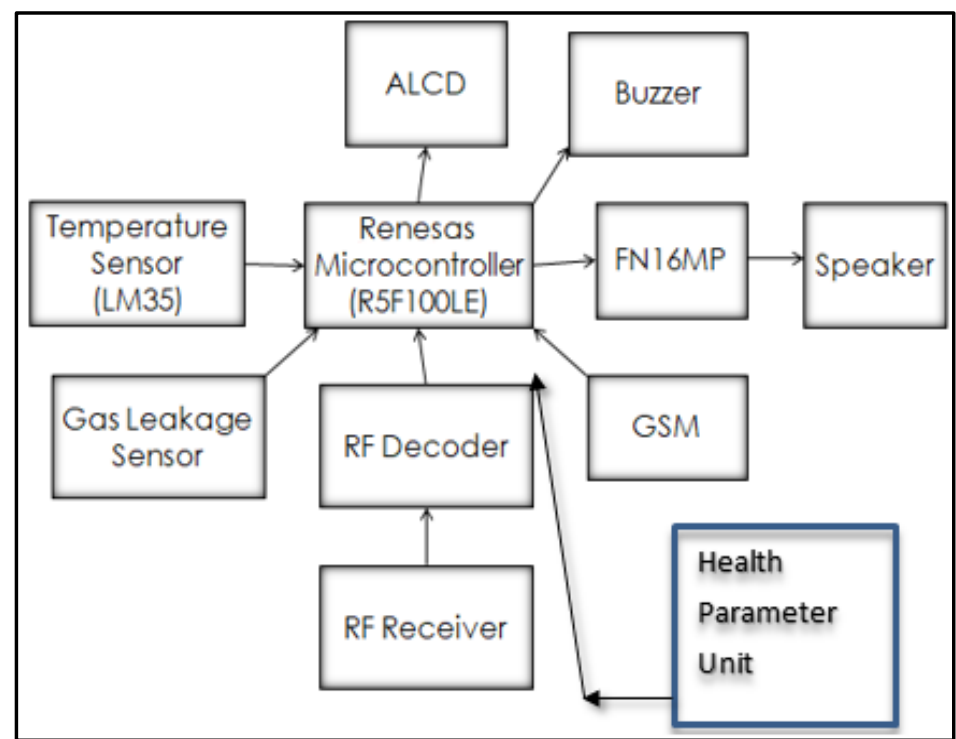

Fig.2: Embedded Unit

As its name proposes, embedded methods something that is joined to something else. An implanted framework can be thought of as a PC equipment framework having programming installed in it. An installed framework has three parts. They are equipment, application programming and Real Time Operating framework (RTOS). This administers the application programming and give system to give the processor a chance to run a procedure according to planning by following an arrangement to control the latencies.

Figure 2 shows the block diagram of the embedded unit which will be installed in the user's house. ALCD displays the messages like: system initialized, GSM active, high temperature, gas leakage, going outside, and so on. The GSM module is used to send alert messages to the user as well as care taker. Alert message is also sent to the nearby emergency unit in case of any accidents like fire in the house.

The FN16MP playback device is used to store the alert messages. A SD card is inserted in the playback device, it is stored with pre-recorded voice outputs. Speakers are connected to this playback device, which plays the voice messages stored in the SD card inserted in the FN16MP playback device. The temperature senor and smoke senor detects high temperature, gas leakage and sends signal to the microcontroller. Which in turn sends signal to the GSM module and playback device to send alert messages to the care concerned. The RF receiver is used to receive RF signal from the touch sensor embedded in the footwear of the user.

\section{B. Slipper unit}

Foot wares of the user are embedded with a touch sensor. This unit uses the wireless communication technology. As soon as the user wears the foot ware, touch sensor senses the pressure further transmitter sends RF signal to the receiver in the embedded unit. Encoded signals are sent to the microcontroller. Which in turn sends signal to the playback module to play the voice output.

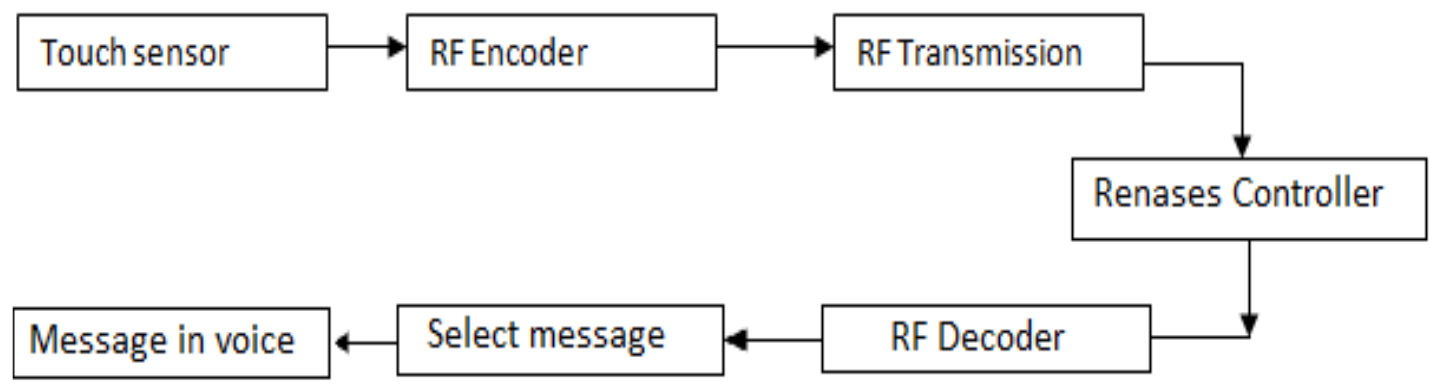

Fig. 3: Slipper Unit 
Vol. 8, Issue 5, May 2019

This voice based assistance reminds the things that the user should take before leaving the house such as spectacle, watch, mobile, walking stick, wallet etc. working phenomenal is as shown in the figure 3 .

\section{Smartphone unit}

Smartphone enables the elderly to communicate with the peers of same age group. It offers chat to share their thoughts and feelings. Using smartphone chat they can plan for get together or it makes them a group of people to engage with. Also it provides health and habit suggest suggesting videos to improve their health and to enjoy with their habit. Drug reminder timings has been set by the care taker using android application and further process is taken by micro controller. Figure 4 depicts the working of smartphone unit.

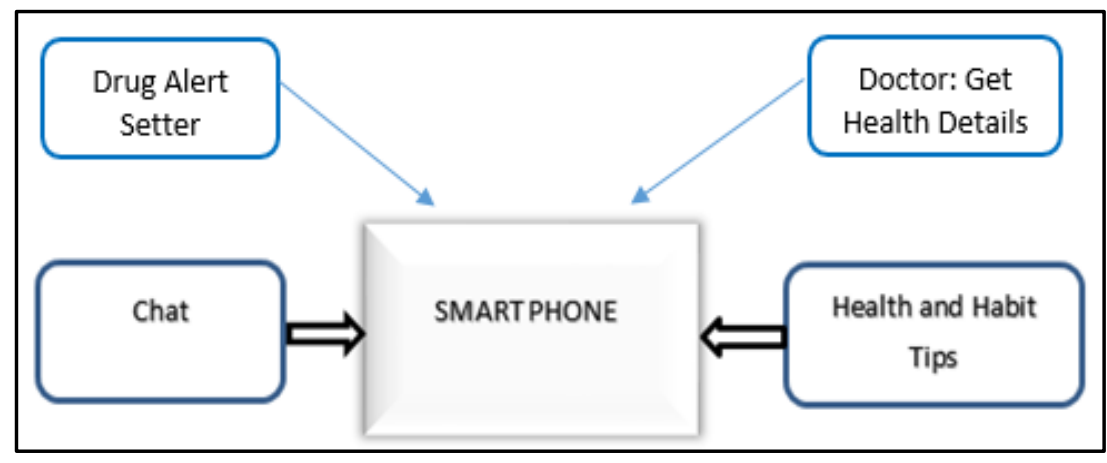

Fig. 4: Smartphone Unit

If there is any health variation then health care personal will get an alert in his android application. It intimates him to give a prescription via email android application. For security reasons he is granted with credentials.

\section{EXPERIMENTAL RESULTS}

Targeted objectives to help the lonely elders are accomplished in the implementation. Figure 5 shows the clumsy structure of embedded unit and slipper units. We can see from the snaps embedded unit contains GSM, Renesas action, playback device and receiver. Controller displays the temperature, smoke and pulse units. It's been checked with the peek value and necessary actions were triggered.

Fig 6 shows the elder chat room typical pages. Once the application is started, it ask for age and name. Once the data is taken, chat room is allowed only to those who are in range of the mentioned age group. Thus social communication for the elder people among same age group is established.

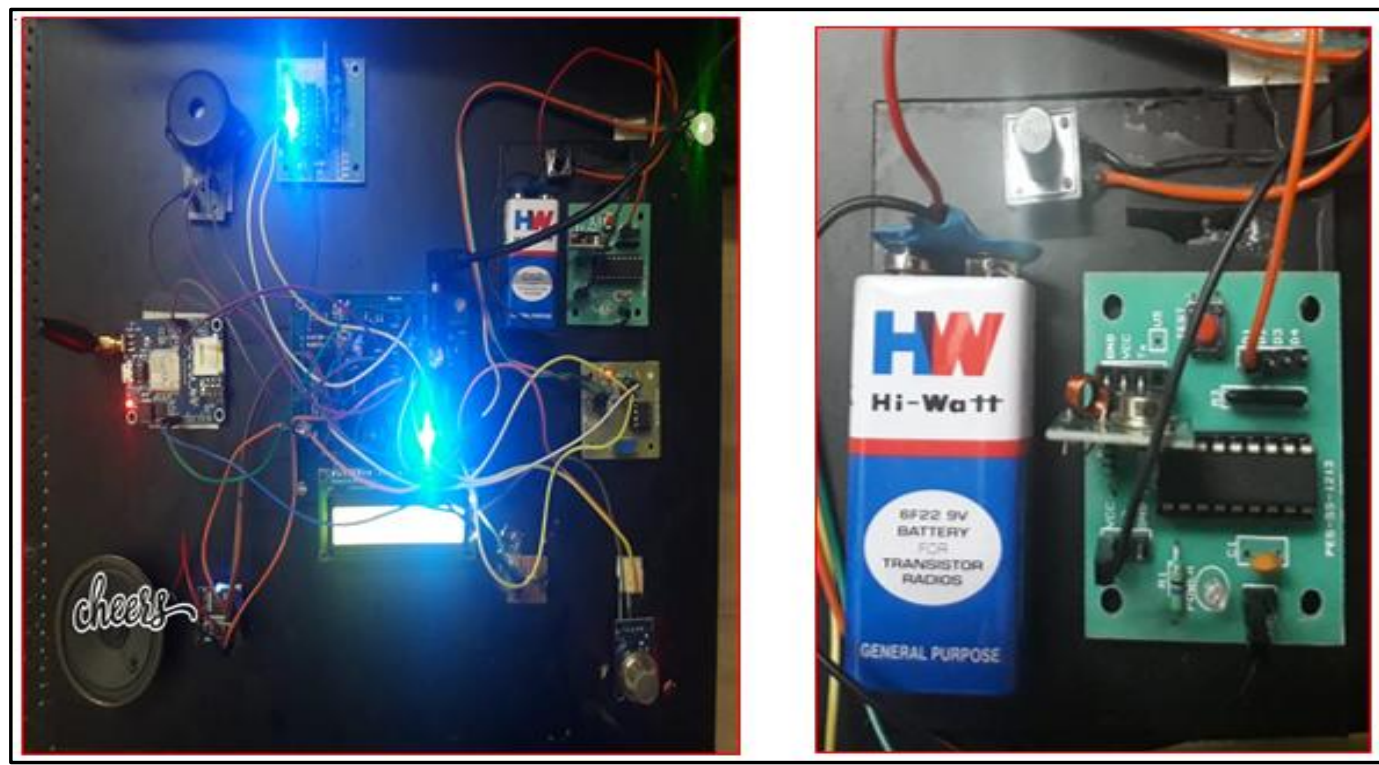

Fig. 5: Embedded and Slipper units 
Vol. 8, Issue 5, May 2019

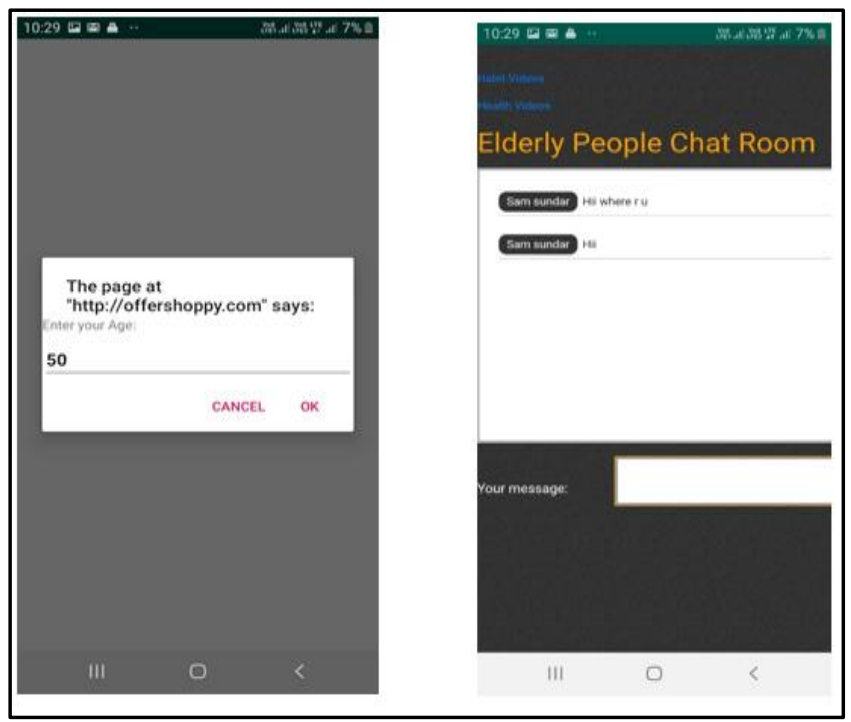

Fig. 6: Elder people chat room

One of the main objective is to remind elder to take meds at prescribed timing. To alert the elder care taker will set the alerting time in his application. When the time matches with the medication time an SMS will be received at elder side. Thus it evokes voice based alert to elderly as shown in the figure 7 . In the figure 7 the second screen shows the mobile number to which message has to be sent. Second row represents the alarm timing need to be set for elder. For security reasons caretaker is provided with credentials.

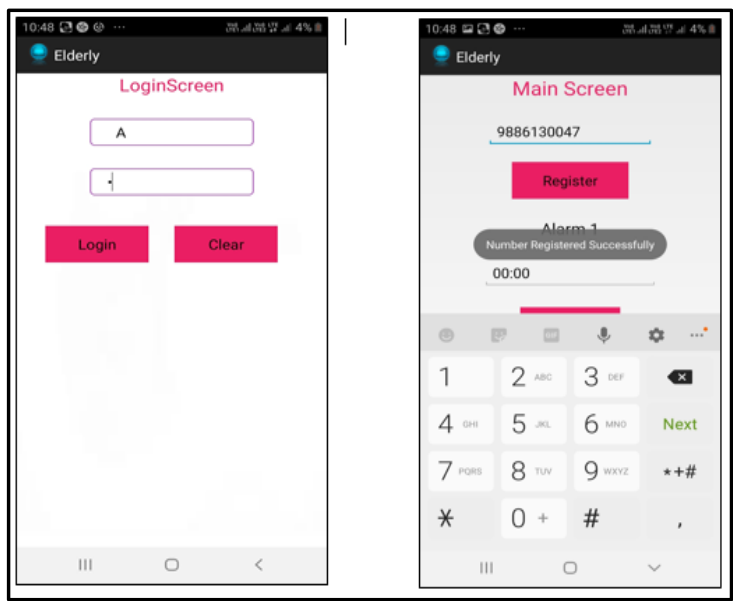

Fig. 7: Drug reminder setter.

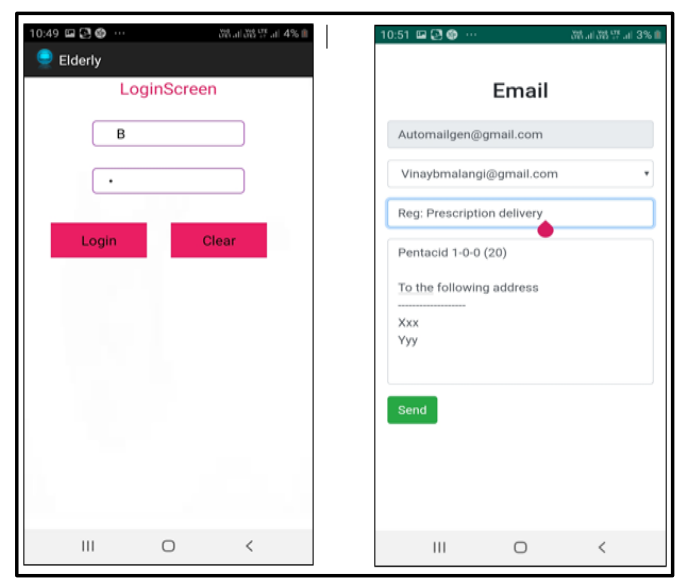

Fig. 8: Prescription to pharmacy for meds.

Through credentials health care personal is allowed view the patient health parameters. If it's found that the health is in abnormal condition he can type the prescription. The prescription is sent to the specified meds outlets via an email as shown in figure 8 . Pharma outlets will take the responsibility of delivering it to the specified elder address. Thus helps in emergency situations.

\section{CONCLUSION}

We Integrated Smartphone technology and technology of Internet of things to build an integrated module which assist the lonely elders and make their life ease and well. Smartphone is a convenient and suitable device due to its rich functionalities. We utilized the smart phone as a frontend or user assistance to elders. Which completely interacts with the Controller and does the necessary actions such as drug intake reminder, to remind forgotten tings, peer communication and the rest. We used renesas controller which is much faster and efficient than the usual IOT technologies such as Audrina, RFID etc. Finally concluding that, the proposed system can greatly help the lonely elders and make their life ease and better in the old age. 
Vol. 8, Issue 5, May 2019

\section{REFERENCES}

[1]. Atika Arshad, Ahmad Fadzil Ismail, Sheroz Khan, Wahidah Hashim and Mohammad Kamrul Hasan, "Bedroom Monitoring System for Isolated Elderly People and Patients", Vol 9(3), 131-137, Asian Journal of Pharmaceutical Research and Health Care, 2017.

[2]. Hyun-Sik Kim, Jong-Su Seo, Jeongwook Seo, “A Daily Activity Monitoring System for Internet of Things Assisted Living in Home Area Networks", Vol. 6, No. 1, International Journal of Electrical and Computer Engineering (IJECE), February 2016.

[3]. Iman Azimi, Amir M. Rahmani, Pasi Liljeberg, Hannu Tenhunen, "Internet of things for remote elderly monitoring: a study from uSsercentered Perspective", Springer-Verlag Berlin Heidelberg 2016

[4]. V. Mighali, L. Patrono, and M. L. Stefanizzi, Joel J. P. C. Rodrigues, Petar Solic, “A Smart Remote Elderly Monitoring System based on IoT Technologies", ICUFN 2017

[5]. Seiichi WATANABE, Megumi ASANO, Tatsuo NAKAZAWA, Ryosuke ANDO, Taro TASAKI, Hiroyuki AOKI," Possibilities of Simple IoT System for Monitoring Elderly People Living Alone", 2017 IEEE 6th Global Conference on Consumer Electronics (GCCE 2017)

[6]. Aslina Baharum, Nurul Hidayah Mat Zain, Aryanto Taharudin, Rozita Hanapepe, Azali Saudi and Rayner Alfred, "Guidelines of User Interface Design for Elderly Mobile Applications: A Preliminary Study", Asian Journal of Information Technology16(1):38-44,January 2017.

[7]. Bin Liu, Zhisheng Yan, and Chang Wen Chen, "Medium Access Control for Wireless Body Area Networks with QoS Provisioning and Energy Efficient Design," IEEE Transactions on Mobile Computing, Vol. 16, No. 2, Feb. 2017, pp. 422-434.

[8]. B. Nandhini and R. Janani, "A smart home monitoring system for elderly people", International Journal of Emerging Technologies in Computational and Applied Sciences, vol. 7, no. 4, pp. 444-447, Feb. 2017

[9]. Aslina Baharum, Nurul Hidayah Mat Zain, Aryanto Taharudin, Rozita Hanapi, Azali Saudi and Rayner Alfred, ”Guidelines of User Interface

[10]. Design for Elderly Mobile Applications: A Preliminary Study", Asian16 (1):38-44, January 2017.

\section{BIOGRAPHIES}

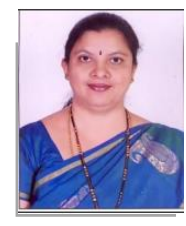

Dr. M C Padma currently working as Professor and Head of the Department Computer science and Engineering, PESCE, Mandya. Has 23 years of experience of in educational institution. She is leading the CSE dept. from past 10 years and involved in various research work. She is awarded with 2 doctorates till now with 6 doctorates under pursuing stage. Sponsored Research work are VGST (Rs.20 Lakhs) -"Establishing the Internet of Things (IoT) Laboratory for Post Graduate and Research programs" and MSME, Govt. of India - "Business Incubator- Support for Entrepreneurial and Managerial Development of SMEs through Incubators". Two more projects which are used for public implementations are "The Smart Unmanned Level Crossing Warning System using GPS and LoRa Protocol” by South Western Railway, Mysore and Projects for Police Department to identify Theft Vehicles. Total no. of papers in referred journals (national and international) is 36 and in conferences (national and international) it is 31. Image Processing and Pattern Recognition, Data mining are the main research areas.

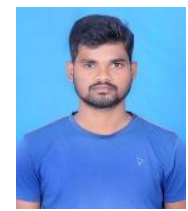

Mr. Vinaya B currently pursuing M.Tech degree from PES College of Engineering, Mandya. Has an industrial experience of 2 years with Tata Consultancy Services Limited, Chennai. Awarded Gold medal from Dr.Ambedkar Institute of Technology, Bangalore, for his academic Excellence in the year 2015 (U.G. - CS\&E). Area of Interests are Internet of Things and AI. 